\title{
A New Fuzzy-based Wavelet Shrinkage Image Denoising Technique
}

\author{
Stefan Schulte $^{1}$, Bruno Huysmans ${ }^{2}$, Aleksandra Pižurica ${ }^{2}$, \\ Etienne E. Kerre ${ }^{1}$ and Wilfried Philips ${ }^{2}$ \\ ${ }^{1}$ Ghent University, Department of Applied Mathematics and Computer Science, \\ Krijgslaan 281 (Building S9), 9000 Gent, Belgium \\ ${ }^{2}$ Ghent University, Dept. of Telecommunications and Information Processing \\ (TELIN), IPI, Sint-Pietersnieuwstraat 41, 9000 Gent, Belgium \\ Email: Stefan.Schulte@UGent.be
}

\begin{abstract}
This paper focuses on fuzzy image denoising techniques. In particular, we investigate the usage of fuzzy set theory in the domain of image enhancement using wavelet thresholding. We propose a simple but efficient new fuzzy wavelet shrinkage method, which can be seen as a fuzzy variant of a recently published probabilistic shrinkage method [1] for reducing adaptive Gaussian noise from digital greyscale images. Experimental results show that the proposed method can efficiently and rapidly remove additive Gaussian noise from digital greyscale images. Numerical and visual observations show that the performance of the proposed method outperforms current fuzzy non-wavelet methods and is comparable with some recent but more complex wavelets methods. We also illustrate the main differences between this version and the probabilistic version and show the main improvements in comparison to it.
\end{abstract}

\section{Introduction}

In general, image denoising imposes a compromise between noise reduction on the one hand and preserving significant image details on the other hand. To achieve a good performance, a noise reduction algorithm should adapt itself to the spatial context. The wavelet transform [2] significantly facilitates the construction of such spatially adaptive algorithms, due to its energy compaction property: it compresses the essential information into a few large coefficients which represent the image details along several resolution scales.

Typical wavelet based denoising methods consist of three steps: (i) compute the discrete wavelet transform (DWT) or a non-decimated wavelet transform, (ii) remove noise from the wavelet coefficients and (iii) reconstruct the enhanced image by using the inverse wavelet transformation. Due to the linearity of the wavelet transform, additive noise in the image domain remains additive in the transform domain as well. If $w_{s, d}(i, j)$ and $y_{s, d}(i, j)$ denote the noisy, respectively

\footnotetext{
2 "A. Pižurica is a postdoctoral researcher of the Fund for the Scientific Research in Flanders (FWO) Belgium"
} 
the noise-free wavelet coefficients of scale $s$ and orientation $d$ then we can model the additive noise in the transform domain as:

$$
w_{s, d}(i, j)=y_{s, d}(i, j)+n_{s, d}(i, j)
$$

where $n_{s, d}(i, j)$ is the corresponding noise component. In this paper we restrict ourselves to additive Gaussian noise.

The second step in the wavelet denoising procedure usually consists of shrinking the wavelet coefficients: the coefficients that contain primarily noise should be reduced to negligable values, while the ones containing a significant noise free component should be reduced less. A common shrinkage approach is the application of simple thresholding nonlinearities to the empirical wavelet coefficients [3-5]: if the coefficient's magnitude is below the threshold $T$ it is reduced to zero, otherwise it is kept or modified. Shrinkage estimators can also result from a Bayesian approach, in which a prior distribution of the noise-free data (e.g., Laplacian [6], generalized Gaussian [7-9], Gaussian Scale Mixture [10]) is integrated in the denoising scheme. The simplest Bayesian methods assume statistically independent data and rely on marginal statistics only $[7,8,11,12]$.

However, algorithms that exploit the different kinds of dependencies between the wavelet coefficients can result in better denoising performance, compared with the ones derived using an independence assumption. The wavelet coefficients are statistically dependent mainly due to two properties of the wavelet transform of natural images: (1) large coefficients will propagate across the scales (interscale dependencies), and (2) if a coefficient is large/small, some of the neighbouring coefficients are also likely to be large/small (intrascale dependencies).

Recently, non-Gaussian bivariate distributions capturing the interscale dependency were proposed[13], and corresponding nonlinear shrinking functions were derived from these distributions using Bayesian estimation theory. Interscale dependencies among the wavelet coefficients are also often modelled with Hidden Markov Trees (HMT)[14,15]. Related methods $[9,16,17]$ use Markov Random Field (MRF) models for capturing intrascale (spatial) dependencies among the wavelet coefficients. It has been proved useful to combine the first order statistical properties of the coefficient magnitudes and their evolution across scales within a joint statistical distribution model [9].

Many other techniques combine inter- and intrascale dependencies. For example, denoising methods based on Gaussian Scale Mixture models, often employ the neighbouring coefficients on the same and adjacent scales [10]. Locally adaptive window-based methods $[1,18]$ are highly performant despite their simplicity. Local contextual HMT models have been developed, which capture both interscale and intrascale information[19,20].

If a certain wavelet coefficient and its neighbouring coefficients are small enough we know that this coefficient is noisy for almost sure and should be put equal to zero. Coefficients above a certain threshold contain the most important image structures and should not be reduced, but coefficients with values around the threshold contain both noise and signals of interest. A good threshold is generally chosen so that most coefficients below the threshold are noise and 
values above the threshold are signals of interest. In such situation it can be advantageous to use fuzzy set theory as kind of soft-threshold method. Fuzzy set theory is a mathematical extension of the binary set theory.

Fuzzy set theory and fuzzy logic [21] offer us powerful tools to represent and process human knowledge represented as fuzzy if-then rules. Fuzzy image processing [22] has three main stages: (i) image fuzzification, (ii) modification of membership values and (iii) image defuzzification. The fuzzification and defuzzification steps are due to the fact that we do not yet possess fuzzy hardware. Therefore, the coding of image data (fuzzification) and decoding of the results (defuzzification) are steps that make it possible to process images with fuzzy techniques. The main power of fuzzy image processing lies in the second step (modification of membership values). After the image data is transformed from input plane to the membership plane (fuzzification), appropriate fuzzy techniques modify the membership values. This can be a fuzzy clustering, a fuzzy rule-based approach, a fuzzy integration approach, etc.

The main advantages of the new method are: (i) the complexity of the method is much lower than the probabilistic one [1] (which results in a lower execution time), (ii) we do not lose any noise reduction performance and (iii) by adding new fuzzy rules it should be easily extendable to incorporate other information as well (e.g. interscale or interband information), to further improve the noise reduction performance (future work).

The paper is structured as follows: In section 2 we discuss the proposed fuzzy shrinkage method. Experimental results are presented in section 3 and section 4 concludes the paper.

\section{Fuzzy Shrinkage Method}

We develop a novel fuzzy wavelet shrinkage method, which is a fuzzy-logic variant of the recent ProbShrink method of [1]. The method of [1] defines for each coefficient $w_{s, d}(i, j)$ two hypotheses: $H_{1}$ : signal of interest present $\left(\left|y_{s, d}(i, j)\right|>\sigma\right)$ and $H_{0}$ : signal of interest absent $\left(\left|y_{s, d}(i, j)\right| \leq \sigma\right)$. The method was named ProbShrink because it shrinks each coefficient according to probability that the coefficient presents a signal of interest given its value $w_{s, d}(i, j)$ and given a local spatial activity indicator $x_{s, d}(i, j)$ as follows: $\hat{y}_{s, d}(i, j)=P\left(H_{1} \mid w_{s, d}(i, j), x_{s, d}(i, j)\right) w_{s, d}(i, j)$. The local spatial activity indicator was defined as the average magnitude of the surrounding wavelet coefficients within a local window. In our notation, this is:

$$
x_{s, d}(i, j)=\frac{\left(\sum_{k=-K}^{K} \sum_{k=-K}^{K}\left|w_{s, d}(i+k, j+l)\right|\right)-\left|w_{s, d}(i, j)\right|}{(2 K+1)^{2}-1}
$$

The method of [1] proceeds by estimating the conditional probability density functions of $w_{s, d}(i, j)$ and $x_{s, d}(i, j)$ given $H_{1}$ and given $H_{0}$ and by using the corresponding likelihood ratios: $\xi\left(w_{s, d}(i, j)\right)=p\left(w_{s, d}(i, j) \mid H_{1}\right) / p\left(w_{s, d}(i, j) \mid H_{0}\right)$ 
and $\eta\left(x_{s, d}(i, j)\right)=p\left(x_{s, d}(i, j) \mid H_{1}\right) / p\left(x_{s, d}(i, j) \mid H_{0}\right)$ and by expressing the shrinkage factor as $\hat{y}_{s, d}(i, j)=\gamma_{s, d}(i, j) /\left(1+\gamma_{s, d}(i, j)\right) w_{s, d}(i, j)$, where $\gamma_{s, d}(i, j)=$ $\rho \xi\left(w_{s, d}(i, j)\right) \eta\left(x_{s, d}(i, j)\right)$ is the generalized likelihood ratio with $\rho=P\left(H_{1}\right) / P\left(H_{0}\right)$.

In this paper, we put the main idea of [1] into a fuzzy logic framework and develop a novel FuzzyShrink method. Namely, we also express the shrinkage factor for the wavelet coefficient $w_{s, d}(i, j)$ as a function of $w_{s, d}(i, j)$ and $x_{s, d}(i, j)$, but instead of estimating the likelihood ratios for these measurements, we impose on them fuzzy membership functions. Our shrinkage factor will also express how likely it is that a coefficient is a signal of interest, but we shall accomplish this by using the appropriate fuzzy norms and co-norms as opposed to the Bayesian formalism and probabilities.

\subsection{Defining Membership Functions and a Fuzzy Rule}

Our reasoning in defining the fuzzy shrinkage rule is the following. If both the neighbourhood around a given position $(i, j)$ and the wavelet coefficient at this position itself $\left(w_{s, d}(i, j)\right)$ contain mainly large (small) coefficients then we have enough indication that we have a signal of interest (noise). If the wavelet coefficient $w_{s, d}(i, j)$ is small but the neighbourhood around a given position $(i, j)$ contains of mainly large coefficients then it is wise to give more importance to the neighbourhood instead wavelet coefficient $w_{s, d}(i, j)$ itself to judge if the value is a signal of interest or not. Otherwise we would give more importance to one single value (that does not correspond to the neighbourhood), which of course is less robust. In this situation (i.e. a small $w_{s, d}(i, j)$ but a large neighbourhood) we should conclude that the position $(i, j)$ is a signal of interest, in spite of the fact that the coefficient is probably lower than the given threshold. This leads us to the Fuzzy Rule 1 introduced below, where the variable $x_{s, d}(i, j)$ represents the average of the wavelet coefficients in the $(2 K+1) \times(2 K+1)$ neighbourhood around a given position $(i, j)$. This variable indicates if the corresponding neighbourhood contains mainly large or small wavelet coefficients.

Fuzzy Rule 1 The definition of the membership degrees in the fuzzy set signal of interest of the wavelet coefficient $w_{s, d}(i, j)$ with scale $s$ and orientation d:

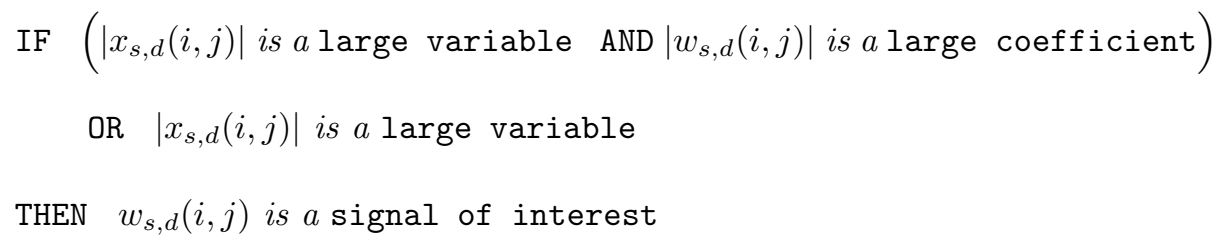

Fuzzy rules are linguistic IF-THEN constructions that have the general form "IF $A$ THEN $B$ ", where $A$ and $B$ are (collections of) propositions containing linguistic variables. $A$ is called the premise or antecedent and $B$ is the consequence of the rule. In Fuzzy Rule 1 we can distinguish two linguistic variables for the consequent: (i) large wavelet coefficients $\left|w_{s, d}(i, j)\right|$ and (ii) large neighbourhood 
values $\left|x_{s, d}(i, j)\right|$. Both linguistic terms are modelled as fuzzy sets. A fuzzy set $C$ [23] in a universe $U$ is characterized by a $U-[0,1]$ mapping $\mu_{C}$, which associates with every element $u$ in $U$ a degree of membership $\mu_{C}(u)$ of $u$ in the fuzzy set $C$. In the following, we will denote the degree of membership by $C(u)$.

The membership functions that are used to represent the two fuzzy sets of (i) large wavelet coefficient $\left|w_{s, d}(i, j)\right|$ and (ii) large neighbourhood value $\left|x_{s, d}(i, j)\right|$, are denoted as $\mu_{w}$ and $\mu_{x}$, respectively. We use triangular membership functions shown in Fig. 1 (a) and (b).

From these figures we see that our method depends on three parameters. As in many image processing methods it is important that each filtering method is adapted to the noise situation (noise level). Therefore we have related all these parameters to the standard deviation of the noise. Good choices for the parameters are: $T_{1}=\sigma, T_{2}=2 \sigma$ and $T_{3}=2.9 \sigma-2.625$, with $\sigma$ the standard deviation of the noise, which is estimated with the median estimator proposed by Donoho and Johnstone [25]. Those threshold values were obtained experimentally by optimising their performance on several test images with several noise levels.

The membership functions for the two fuzzy sets that are shown in Fig. 1 function as a kind of lookup-tables for the likelihood ratios of the probabilistic versions [1].

In Fuzzy Rule 1 we can observe an intersection and a union of two fuzzy sets. The intersection $A \cap B$ of two fuzzy sets $A$ and $B$ is generally specified by a binary mapping $D$ leading to: $(A \cap B)(y)=D(A(y), B(y))$. The union $A \cup B$ of two fuzzy sets $A$ and $B$ is specified by a binary mapping $S$ leading to: $(A \cup$ $B)(y)=S(A(y), B(y)$ ).In fuzzy logic, triangular norms (roughly the equivalent of AND operations) and triangular co-norms (roughly the equivalent of OR operations) are used to represent the intersection and the union of two fuzzy sets, respectively. Some well-known triangular norms together with their dual triangular co-norm are shown in Table 1. From all possible triangular norms the minimum norm is the largest and the weak norm (Table 1) is the smallest. From all possible triangular conorms the strong norm is the largest and the maximum norm (Table 1) is the smallest. We have chosen for a t-norm (with his dual conorm) which is situated between those two extremes, i.e. the product and the probabilistic sum, respectively. So the antecedent $\left(\left|x_{s, d}(i, j)\right|\right.$ is large variable AND $\left|w_{s, d}(i, j)\right|$ is large coefficient $)$ can be translated into the "truth" value: $\mu_{x}\left(\left|x_{s, d}(i, j)\right|\right) \cdot \mu_{w}\left(\left|w_{s, d}(i, j)\right|\right)$, where $\mu_{x}$ and $\mu_{w}$ are the membership functions for the fuzzy set large variables and large coef ficient, respectively. In the next subsection we explain how to shrink the wavelet coefficients of a noisy image.

\subsection{Output of the Method}

The shrinkage rule of the proposed method for scale $s$, direction $d$ and position $(i, j)$ is calculated as follows:

$$
\hat{y}_{s, d}(i, j)=\gamma\left(w_{s, d}(i, j), x_{s, d}(i, j)\right) \cdot w_{s, d}(i, j)
$$




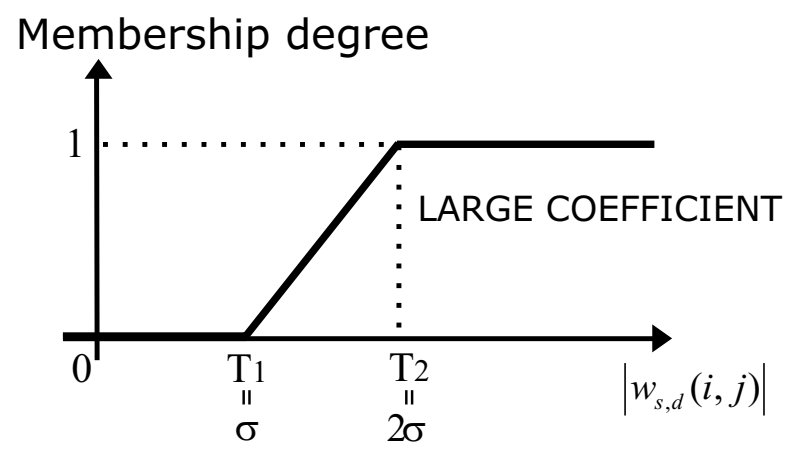

(a)

Membership degree

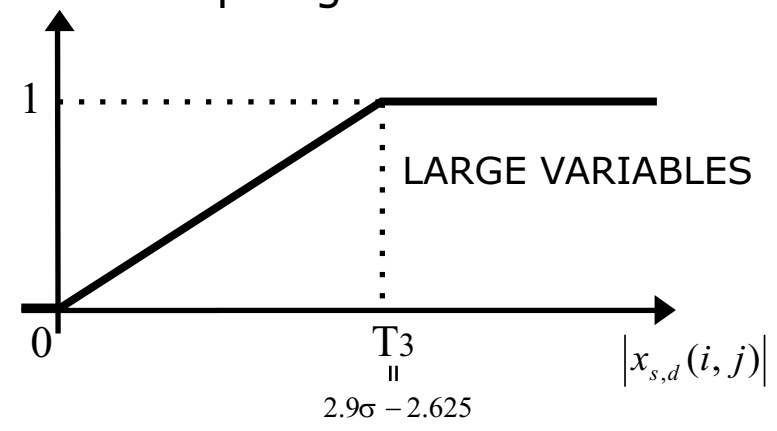

(b)

Fig. 1. (a) The membership function LARGE COEFFICIENT denoted as $\mu_{w}$ for the fuzzy set large coefficient and (b) The membership function LARGE VARIABLE denoted as $\mu_{x}$ for the fuzzy set large variable. 
Table 1. Some well-known triangular norms ( $T$-norms) and triangular conorms $(T$ conorms)

\begin{tabular}{|c|c|}
\hline \multicolumn{2}{|r|}{$\overline{T \text {-norms }}$} \\
\hline minimum & $\min (x, y)$ \\
\hline algebraic product & $x \cdot y$ \\
\hline weak & $\begin{cases}\min (x, y) & \text { if } \max (x, y)=1 \\
0 & \text { otherwise }\end{cases}$ \\
\hline bounded sum & $\max (0, x+y-1)$ \\
\hline \multicolumn{2}{|r|}{$\overline{T \text {-conorms }}$} \\
\hline maximum & $\max (x, y)$ \\
\hline probabilistic sum & $x+y-x \cdot y$ \\
\hline strong & $\begin{cases}\max (x, y) & \text { if } \min (x, y)=0 \\
1 & \text { otherwise }\end{cases}$ \\
\hline bounded sum & $\min \left(1, \mu_{A}(x)+\mu_{B}(x)\right)$ \\
\hline
\end{tabular}


with $\hat{y}_{s, d}(i, j)$ the shrink output coefficient for scale $s$, direction $d$ and position $(i, j)$ and where $\gamma\left(w_{s, d}(i, j), x_{s, d}(i, j)\right)$ is the degree of activation of Fuzzy Rule 1 for the wavelet coefficient $w_{s, d}(i, j)$. This value indicates the membership degree in the fuzzy set signal of interest for the wavelet coefficient $w_{s, d}(i, j)$. If the membership degree has value 1 , this means that the corresponding coefficient is a signal of interest certainly (and should not be changed), while a degree zero indicates that the coefficient is certainly not a signal of interest (and should be set equal to zero). A value between zero and one indicates that we do not know quite sure if this coefficient is a signal of interest or not. This means that the coefficient is a signal of interest only to a certain degree. The calculation of the value $\gamma\left(w_{s, d}(i, j), x_{s, d}(i, j)\right)$ is illustrated in expression (4).

$$
\begin{aligned}
\gamma\left(w_{s, d}(i, j), x_{s, d}(i, j)\right) & =\alpha+\mu_{x}\left(\left|x_{s, d}(i, j)\right|\right)-\alpha \cdot \mu_{x}\left(\left|x_{s, d}(i, j)\right|\right) \\
\text { with } \quad \alpha & =\mu_{x}\left(\left|x_{s, d}(i, j)\right|\right) \cdot \mu_{w}\left(\left|w_{s, d}(i, j)\right|\right)
\end{aligned}
$$

Actually, the $\alpha$ of expression (4) can be seen as the fuzzy counterpart of generalized likelihood ratio used in the probabilistic version [1]. One can see that we used the product and probabilistic sum for the triangular norm and co-norm, respectively.

\section{Experimental Results}

In this section we present some experimental results. We compared our new fuzzy wavelet-based shrinkage method with (i) other well-known fuzzy filters and (ii) recently developed wavelet-based methods. More precisely we have:

- FUZZY: the GOA filter [26], FRINRM [27] (fuzzy randomly valued impulse noise reduction method), HAF [28] (histogram adaptive fuzzy), EIFCF [29] (extended iterative fuzzy control based filter), SFCF [29] (smoothing fuzzy control based filter), DWMAV [30] (decreasing weight fuzzy filter with moving average centre), GMAV [30] (Gaussian fuzzy filter with moving average centre), AFSF [31] (the adaptive fuzzy switching filter), FSB [32,33] (fuzzy similarity filter) and AWFM [34,35] (adaptive weighted fuzzy mean).

- WAVELET: the bivariate wavelet shrinkage function proposed by Şendur [36], the feature-based wavelet shrinkage method proposed by Balster [37] and the probabilistic shrinkage function proposed by Pižurica [1].

We have used a redundant wavelet transform with the Haar wavelet and four resolution scales and a neighbourhood of size $9 \times 9(K=4)$ for both the probabilistic version and the proposed one. As a measure of objective dissimilarity between a filtered image $F$ and the original noisefree one $O$, we use the peak signal to noise ratio (PSNR).

In order to get a clear idea of the performance of all mentioned methods we have carried out experiments for three well known test images: 'Lena', 'Peppers' 
and 'Barbara', each of size $512 \times 512$. The numerical results for the corrupted versions (for $\sigma=5,20,30$ and 40) are shown in Table 2. From this Table we can make the following conclusions:

- The wavelet-based methods perform generally better than the state of the art fuzzy non-wavelet based methods for the additive noise type. Waveletbased methods reduce the noise quite well for both low and high $\sigma$ values, while the fuzzy-based methods only perform well for higher noise levels.

- The only fuzzy-based method that receives comparable results to the wavelet ones is the GOA filter. This filter even results in the best PSNR value for the Peppers images corrupted with $\sigma=30$ and 40 additive Gaussian noise. But the GOA filter is developed only for a specific group of images like the Lena and the Peppers images. If an image contains regions with lots of fine details, texture or contours (like grass, hair etc.) then the GOA filter destroys such structures, which is confirmed by the low PSNR value for the Barbara image.

- Generally, the best numerical results were received by the proposed and the probabilistic shrinkage method. The proposed fuzzy shrinkage method performs quite similar as the probabilistic one.

The visual performance of the best numerical filters is given in Fig. 2, where we show the denoised versions of the Barbara image corrupted with $\sigma=40$ additive Gaussian noise. It is shown that the proposed and the probabilistic shrinkage method do not only yield the highest PSNR values (Table 2), but also the best visual results. The other wavelet-based methods reduce the noise well but introduce typical wavelet compression artefacts. From Fig. 2 (f) we see that the GOA filter destroys more images structures than the wavelet-based method, which results in a blurrier image. We can also conclude that the other state of the art fuzzy-based methods are not able to receive such good visual performances as the wavelet-based methods.

Previous experiments have clearly confirmed that the proposed method performs at least as well as the probabilistic method of [1]. In this paragraph we will illustrate that the proposed method, which can be viewed at

http://www.fuzzy.ugent.be/ACIVS06.html, has a lower complexity than the probabilistic version. In Table 3 we have compared the execution time between those two methods for the noise reduction of one wavelet band of size $512 \times 512$. The comparison is done by implementing both methods in the same programming language namely Java (not Matlab because Matlab uses many C-files so that the comparison would not be correct). The main difference of both methods is that the probabilistic method has to estimate the (image dependent) distributions first before the filtering can be started while the fuzzy shrinkage method can be applied directly. This fuzzy shrinkage method uses membership functions that are shown in Fig. 1, which functions as a kind of lookup-tables for the likelihood ratios of the probabilistic versions [1]. This explains while the proposed method is less complex. The execution time for the distribution estimation of [1] does not depend on the used neighbourhood size. Next even if we observe the execution 
Table 2. PSNR results for the $(512 \times 512-)$ Lena, Peppers and Barbara images corrupted with additive Gaussian noise with $\sigma=5, \sigma=20, \sigma=30$ and $\sigma=40$ and several fuzzy and wavelet based denoising methods.

\begin{tabular}{|c|c|c|c|c|c|c|c|c|c|c|c|c|}
\hline & \multicolumn{4}{|c|}{ Lena } & \multicolumn{4}{|c|}{ Peppers } & \multicolumn{4}{|c|}{ Barbara } \\
\hline $\bar{\sigma}$ & 5 & 20 & 30 & 40 & 5 & 20 & 30 & 40 & 5 & 20 & 30 & 40 \\
\hline Noisy & 34.2 & 22.1 & 18.6 & 16.1 & 34.2 & 22.1 & 18.6 & 16.1 & 34.2 & 22.1 & 18.6 & 16.1 \\
\hline New & 38.2 & 32.4 & 30.5 & 29.2 & 37.1 & 32.0 & 30.5 & 29.3 & 37.2 & 29.7 & 27.5 & 25. \\
\hline ProbShrink & 38.3 & 32.3 & 30.4 & 29.2 & 7.1 & 32.0 & 30.5 & 29.3 & 37.2 & 29.4 & 27.1 & 25.5 \\
\hline BiShrink & 37.4 & 31.2 & 29.3 & 28.1 & 35.7 & 31.0 & 29.3 & 28.1 & 36.2 & 28.2 & 26.1 & 24.8 \\
\hline Balster & 37.2 & 31.5 & 29.8 & 28.5 & 34.4 & 31.7 & 30.1 & 28.9 & 35.8 & 27.6 & 25.3 & 24.0 \\
\hline GOA & 36.4 & 31.2 & 29.5 & 28. & 35.6 & 31.7 & 30.0 & 28.6 & 33.9 & 25.8 & 24.2 & 23.5 \\
\hline FRINRM & 34.9 & 26.2 & 23.8 & 21.3 & 4.3 & 25.4 & 22.2 & 20.4 & 4.2 & 23.7 & 21.4 & 20.2 \\
\hline HAF & 33.7 & 29.5 & 26.9 & 24.8 & 33.1 & 28.8 & 26.2 & 24.0 & 25.3 & 24.4 & 23.3 & 21.1 \\
\hline EIFCF & 33.6 & 29.3 & 27.2 & 25.5 & 33.8 & 29.5 & 27.3 & 25.6 & 25.5 & 24.6 & 23.7 & 22.8 \\
\hline SFCF & 33.1 & 29.4 & 26.2 & 23.5 & 33.1 & 29.4 & 26.3 & 23.6 & 25.8 & 24.8 & 23.3 & 21.6 \\
\hline DWMAV & 33.2 & 29.6 & 27.2 & 25.2 & 32.9 & 29.4 & 27.1 & 25.1 & 25.2 & 24.4 & 23.5 & 22.6 \\
\hline GMAV & 33.5 & 29.3 & 26.7 & 24.7 & 33.3 & 29.4 & 26.8 & 24.7 & 25.1 & 24.3 & 23.3 & 22.2 \\
\hline AFSF & 34.5 & 27.6 & 25.0 & 23.0 & 34.4 & 27.6 & 24.9 & 22.9 & 26.0 & 23.9 & 22.5 & 21.2 \\
\hline & 33.8 & 28.8 & 25.5 & 23.1 & 33.7 & 28.9 & 25.7 & 23.3 & 25.2 & 23.9 & 22.6 & 21.2 \\
\hline AWFM & 34.3 & 29.2 & 26.1 & 22.1 & 34.2 & 29.4 & 25.2 & 23.0 & 26.1 & 24.5 & 22.9 & 22.9 \\
\hline
\end{tabular}

time of the denoising methods only we see that the fuzzy shrinkage method is faster. This small difference is analysed in Table 4, where we have compared the amount of operations that have to be carried out to perform the denoising method for one wavelet band only. We observe that the amount of logical operations is very similar. But if we know that memory operations cause more time to be done than all other operations we see why the probabilistic method is slower.

In Fig. 3 we have illustrated the mean execution time of both methods (for a neighbourhood size of $5 \times 5$ and $9 \times 9$ (i.e. $K=2$ and $K=4$, respectively)) and the fuzzy non-wavelet based GOA filter [26]. We observe that the non-wavelet based method GOA performs much faster than the two wavelet based algorithms. The main two reasons for this difference are (i) in wavelet-based methods, the images have to be transformed into the wavelet domain and (ii) for both methods we have used a redundant wavelet transformation, so that the amount of data becomes larger. The second observation that can be made from Fig. 3 is that the proposed method is significantly faster than the probabilistic shrinkage method, which confirms that the proposed method is less complex than the probabilistic one.

\section{Acknowledgment}

This work was financially supported by the GOA-project 12.0515 .03 of Ghent University. 


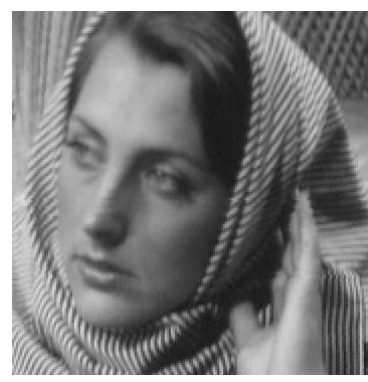

(a)

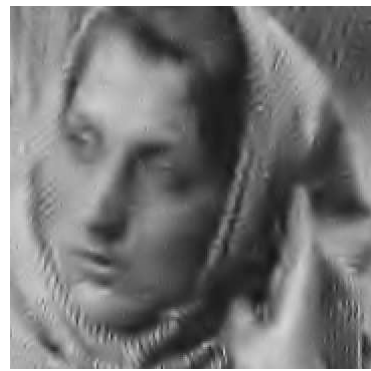

(d)

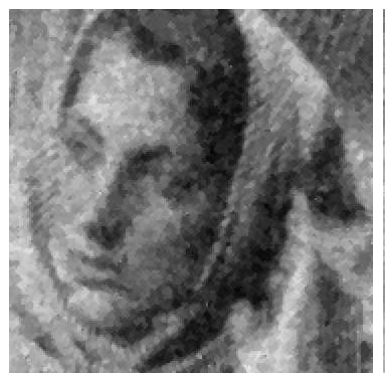

(g)

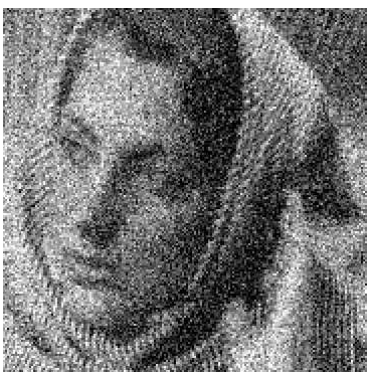

(b)

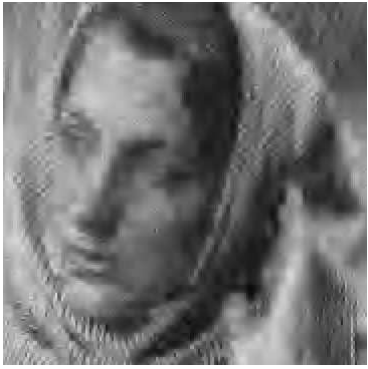

(e)

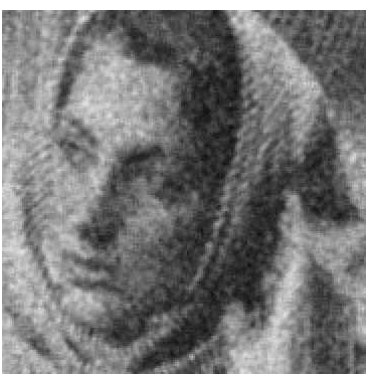

(h)

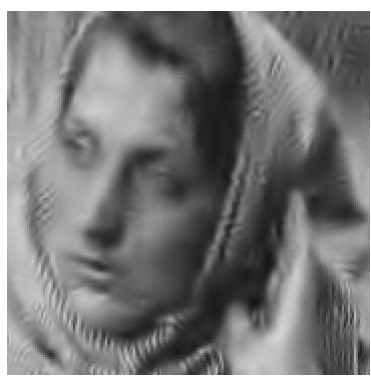

(c)

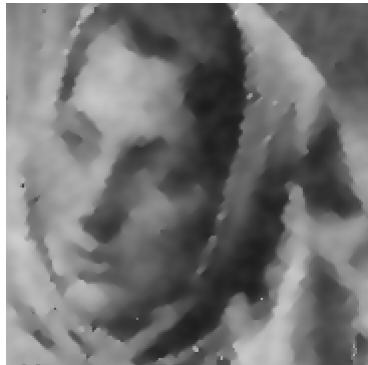

(f)

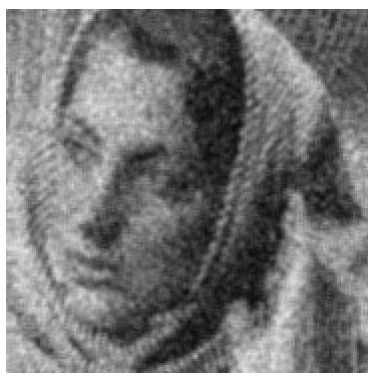

(i)

Fig. 2. The restoration of a magnified part of the Barbara image corrupted with additive Gaussian noise $(\sigma=40)$ : (a) the noise free part (b) the noisy part (c) the proposed filter, (d) ProbShrink, (e) BiShrink, (f) GOA, (g) EIFCF (h), HAF, (i) DMWAV. 
Table 3. Comparison between the proposed fuzzy shrinkage method (FuzzyShrink) and the probabilistic shrinkage method (ProbShrink) in term of the execution time $(\mathrm{ms})$ for the denoising method of a noisy wavelet band of size $(512 \times 512)$.

\begin{tabular}{|c|c|rrrr|}
\hline & & \multicolumn{4}{|c|}{ Execution time in $m s$} \\
\hline & & $K=1$ & $K=2$ & $K=3$ & $K=4$ \\
\hline FuzzyShrink & Total & 58.5 & 108.0 & 179.3 & 273.5 \\
\hline \hline ProbShrink & Denoising & 63.4 & 110.6 & 195.8 & 282.0 \\
& Distribution estimation & 179.9 & 180.0 & 180.3 & 180.3 \\
\cline { 2 - 6 } & Total & 243.3 & 290.6 & 376.1 & 462.3 \\
\hline
\end{tabular}

Table 4. Comparison between the proposed fuzzy shrinkage method (FuzzyShrink) and the probabilistic shrinkage method (ProbShrink) of the amount of operations necessary for the denoising methods of a noisy wavelet band of size $(N \times M)$ with $\eta=M \cdot N$ (exclusive the amount of operations necessary to calculate the distribution estimation).

\begin{tabular}{|c|ccccc|}
\hline & \multicolumn{5}{|c|}{ Execution time in ms } \\
\hline \multirow{3}{*}{ FuzzyShrink } & + & - & $/$ & $*$ & memory \\
\cline { 2 - 6 } ProbShrink & $\left(4+(2 K+1)^{2}\right) \eta$ & $6 \eta$ & $3 \eta$ & $4 \eta$ & $\left((2 K+1)^{2}\right) \eta$ \\
& $\left(5+(2 K+1)^{2}\right) \eta$ & $\eta$ & $2 \eta$ & $5 \eta$ & $\left((2 K+1)^{2}+3\right) \eta$ \\
\hline
\end{tabular}
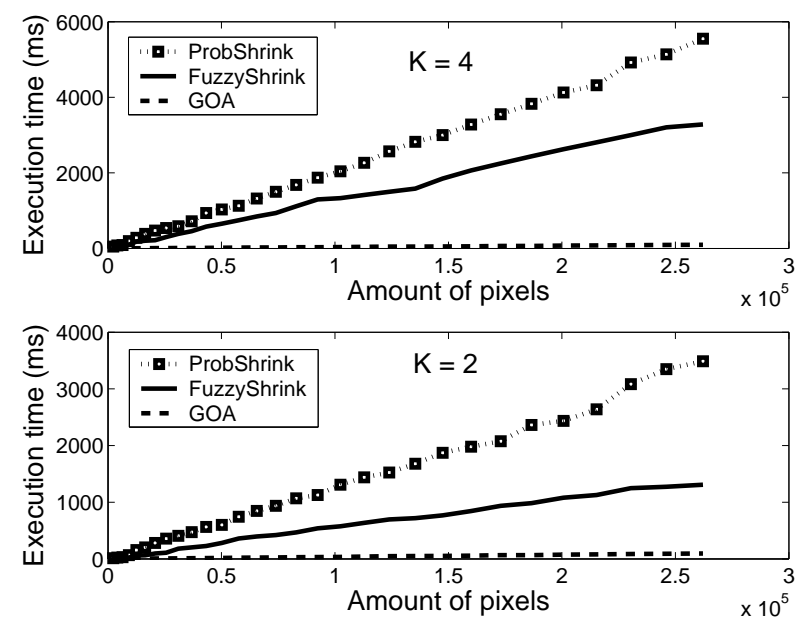

Fig. 3. Comparison of the mean execution time for the ProbShrink method of [1], the GOA filter of [26] and the proposed FuzzyShrink method with (a) a windowsize $K=2$ and (b) a windowsize $K=4$.

\section{Conclusion}

In this paper an alternative wavelet based soft-computing method for the recently published probabilistic shrinkage method of Pižurica [1] for the reduction 
of additive Gaussian noise in digital images was proposed. Experimental results show that the proposed method receives the same noise reduction performance as the probabilistic one, which outperforms the current fuzzy-based algorithms and some recently published wavelet-based methods. Next we have shown that the proposed method clearly reduces the complexies of the probabilistic shrinkage method in terms of execution time. A future advantage of the method is the ability of incorporate more information (e.g. interscale and/or colour information) by adding other fuzzy rules to improve the noise reduction performance. Future work should be done on this promising issue.

\section{References}

1. Pižurica, A., Philips, W.: Estimating the probability of the presence of a signal of interest in multiresolution single- and multiband image denoising. IEEE Transactions on Image Process. 15(3) (2006) 654-665

2. Resnikoff, H. L., Wells, R.O.: Wavelet Analysis: The Scalable Structure of Information Springer-Verlag (1998)

3. Donoho, D.: Denoising by soft-thresholding. IEEE Transactions on Information Theory. 41(5) (1995) 613-627

4. Donoho, D., Johnstone, I.: Adapting to unknown smoothness via wavelet shrinkage. Journal of the American Statistical Association. 90 (1995) 1200-1224

5. Chang, S., Yu, B., Vetterli, M.: Adaptive wavelet thresholding for image denoising and compression. IEEE Transactions on Image Processing. 9(9) (2000) 1532-1546

6. Hansen, M., Yu, B.: Wavelet thresholding via mdl for natural images. IEEE Transactions on Information Theory. 46(8) (2000) 1778-1788

7. Simoncelli, E., Adelson, E.: Noise removal via Bayesian wavelet coring. Proceedings IEEE International Conference on Image Processing (ICIP'96). Lausanne, Switserland (1996) 379-382

8. Moulin, P., Liu, J.: Analysis of multiresolution image denoising schemes using generalized gaussian and complexity priors. IEEE Transactions on Information Theory. 45(4) (1999) 909-919

9. Pižurica, A., Philips, W., Lemahieu, I., Acheroy, M.: A joint inter- and intrascale statistical model for Bayesian wavelet based image denoising. IEEE Transactions on Image Processing. 11(5) (2002) 545-557

10. Portilla, J., Strela, V., Wainwright, M, Simoncelli, E.: Image denoising using gaussian scale mixtures in the wavelet domain. IEEE Transactions on Image Processing. 12(11) (2003) 1338-1351

11. Vidakovic, B.: Nonlinear wavelet shrinkage with bayes rules and bayes factors. Journal of the American Statistical Association. 93 (1998) 173-179

12. Chipman, H., Kolaczyk, E., McCulloch, R.: Adaptive Bayesian wavelet shrinkage. Journal of the American Statistical Association. 92 (1997) 1413-1421

13. Sendur, L., Selesnick, I.: Bivariate shrinkage functions for wavelet-based denoising exploiting interscale dependency. IEEE Transactions on Signal Processing. 50(11) (2002) 2744-2756

14. Crouse, M., Nowak, R., Baranuik, R.: Wavelet-based statistical signal processing using hidden Markov models. IEEE Transactions on Signal Processing. 46(4) (1998) 886-902 
15. Romberg, J., Choi, H., Baraniuk, R.: Bayesian tree-structured image modeling using wavelet-domain hidden markov models. IEEE Transactions on Image Processing. 10 (2001) 1056-1068

16. Malfait, M., Roose, D.: Wavelet-based image denoising using a markov random field a priori model. IEEE Transactions on Image Processing. 6(4) (1997) 549-565

17. Jansen, M., Bultheel, A.: Empirical Bayes approach to improve wavelet thresholding for image noise reduction. Journal of the American Statistical Association. 96(454) (2001) 629-639

18. Mihcak, M., Kozintsev, I., Ramchandran, K., Moulin, P.: Low complexity image denoising based on statistical modeling of wavelet coefficients. IEEE Signal Processing Letters. 6 (1999) 300-303

19. Fan, G., Xia, X.: Image denoising using local contextual hidden markov model in the wavelet domain. IEEE Signal Processing Letters. 8(5) (2001) 125-128

20. Fan, G., Xia, X.: Improved hidden Markov models in the wavelet domain. IEEE Transactions on Signal Processing. 49) (2001) 115-120

21. Kerre, E.E.: Fuzzy sets and approximate Reasoning. Xian Jiaotong University Press (1998).

22. Tizhoosh, H.R.: Fuzzy-Bildverarbeitung: Einfhrung in Theorie und Praxis. Springer-Verlag (1997)

23. Zadeh, L.A.: Fuzzy Sets. Information and Control 8(3) (1965) 338-353

24. Zadeh, L. A.: Fuzzy logic and its application to approximate reasoning. Information Processing 74 (1973) 591-594

25. Donoho, D.L., Johnstone, I.M.: Ideal spatial adaptation by wavelet shrinkage. Biometrika 81 (1994) 425-455

26. Van De Ville, D., Nachtegael, M., Van der Weken, D., Kerre, E.E., Philips, W.: Noise reduction by fuzzy image filtering. IEEE Transactions on Fuzzy Systems 11(4) (2003) 429-436

27. Schulte, S., De Witte, V., Nachtegael, M., Van der Weken, D., Kerre, E.E.: Fuzzy Random Impulse Noise Reduction Method. Fuzzy Sets and Systems (2006) (submitted)

28. Wang, J.H., Chiu, H.C.: An adaptive fuzzy filter for restoring highly corrupted images by histogram estimation. Proceedings of the National Science Council Part A 23 (1999) 630-643

29. Farbiz, F., Menhaj, M.B., Motamedi, S.A.: Edge Preserving Image Filtering based on Fuzzy Logic. Proceedings of the 6th EUFIT conference (1998) 1417-1421

30. Kwan, H.K., Cai, Y.: Fuzzy filters for image filtering. Proceedings of Circuits and Systems (MWSCAS-2002). The $200245^{\text {th }}$ Midwest Symposium (2002) III-672-5.

31. Xu, H., Zhu, G., Peng, H., Wang, D.: Adaptive fuzzy switching filter for images corrupted by impulse noise. Pattern Recognition Letters 25 (2004) 1657-1663

32. Tolt, G., Kalaykov, I.: Fuzzy-similarity-based Noise Cancellation for Real-time Image Processing. Proceedings of the 10th IEEE International Conference on Fuzzy Systems (FUZZ-IEEE) 1 15 -18

33. Tolt, G., Kalaykov, I.: Fuzzy-Similarity-Based Image Noise Cancellation. Lecture Notes in Computer Science: Advances in Soft Computing (AFSS 2002) 2275 (2002) 408-413

34. Lee, C.S., Kuo, Y.H., Yu, P.T.: Weighted Fuzzy Mean Filters for Image Processing. Fuzzy Sets and Systems 89(2) (1997) 157-180

35. Kuo, Y.H., Lee, C.S., Chen, C.L.: High-stability AWFM filter for signal restoration and its hardware design. Fuzzy Sets and Systems 114(2) (2000) 185-202

36. Şendur, L., Selesnick, I.W.: Bivariate Shrinkage Functions for Wavelet-based Image Denoising. IEEE Transactions on Signal Processing 50(11) (2002) 2744-2756 
37. Balster, E.J., Zheng, Y.F., Ewing, R.L.: Feature-based wavelet shrinkage algorithm for image denoising. IEEE Transactions on Image Process. 14(3) (2005) 2024-2039 